\title{
Breast Cancer Screening Participation of Women with Chronic Diseases in Korea: Analysis of the 2012 Korean National Health and Nutrition Examination Survey
}

\author{
Laurence Twizeyimana, Yeol Kim*
}

\begin{abstract}
Chronic disease morbidity is a concern for cancer research. Contradictory results have been reported concerning adherence to breast cancer screening among patients with chronic diseases. The study was conducted to assess the adherence to breast cancer screening among women with chronic diseases in Korea. It was a cross sectional and population based study; the data came from the Korean National Health and Nutrition Examination Survey (KNHANES) 2012. Participation in breast cancer screening was analyzed among women who had at least one of eight chronic diseases (hypertension, diabetes, cancer, dyslipidemia, stroke, depression, osteoarthritis and asthma). Pearson's chi-squared test and multiple logistic regression analysis were performed using STATA version 14. A total 2,404 women aged 40 years or older were included in analysis. Among them, $77.3 \%$ had experienced breast cancer screening. In logistic regression model, adherence to breast cancer screening was lower in women who have ever been diagnosed as diabetes mellitus (odds ratio $(\mathrm{OR})=0.47,95 \%$ confidence interval $(95 \% \mathrm{CI})=0.31-0.72)$, asthma $(\mathrm{OR}=0.44,95 \% \mathrm{CI}=0.24-0.82)$ and higher in women who have ever been diagnosed as dyslipidemia $(\mathrm{OR}=1.85,95 \% \mathrm{CI}=1.27-2.69)$, osteoarthritis $(\mathrm{OR}=$ $1.42,95 \% \mathrm{CI}=1.31-2.06)$ and cancers $(\mathrm{OR}=2.70,95 \% \mathrm{CI}=1.26-5.79)$ compared to women without those chronic diseases. Based on treatment of chronic diseases, lower participation in breast cancer screening was observed in women who were on treatment of diabetes mellitus $(\mathrm{OR}=0.48,95 \% \mathrm{CI}=0.31-0.74)$, asthma $(\mathrm{OR}=0.41,95 \% \mathrm{CI}=0.15-0.92)$ and stroke $(\mathrm{OR}=0.37,95 \% \mathrm{CI}=0.14-0.97)$, and higher in women who were on treatment of dyslipidemia $(\mathrm{OR}=1.37$, $95 \% \mathrm{CI}=1.42-2.14)$. In conclusion, low participation rate in breast cancer screening in women with some chronic diseases was identified. The results from this study may provide an important contribution for helping to maintain and increase participation in cancer screening of patients with chronic diseases.
\end{abstract}

Keywords: Breast cancer- screening- mammography- participation- chronic disease

Asian Pac J Cancer Prev, 20 (1), 207-213

\section{Introduction}

Cancer is a leading cause of death, and cancer burden is predictable to grow worldwide due to aging population. For 2012, GLOBOCAN reported 14.1 million new cancer cases and 8.2 million deaths from cancer worldwide (Ferlay et al., 2015). Cancer screening remains essential in cancer detection at early stage before the cancer gets complicated as it increases the chance for successful treatment through early diagnosis (Smith et al., 2006).

For breast cancer control, although the percentage of mortality reduction attributable to screening has been subject to debate (Autier et al., 2011). Recent studies have found a $10 \%$ to $20 \%$ reduction in breast cancer mortality among women who underwent mammographic screening (Autier et al., 2011; Tabar et al., 2011). Scientific evidence showed that screening for either breast cancer in appropriate age group reduces mortality by $20 \%$ to 60
$\%$ depending on the condition and baseline risk-level of the group being screened (Kerlikowske et al., 1995; La Vecchia et al., 1984).

Chronic illness is an important of increasing implication for cancer research worldwide (Wild, 2012). Comorbidity is common among cancer patients and with an aging population is becoming more (Sarfati et al., 2016). Comorbidity possibly affects the development, stage at diagnosis, treatment, and outcomes of people with cancer. Though, chronic conditions are already the leading cause of death worldwide and their burden is expected to increase, it has been shown that all-cause mortality as well as cancer specific mortality is higher for recently diagnosed cancer patients suffering from chronic conditions, even when stage at diagnosis or treatment are taken into description (Mendis et al., 2015; Piccirillo et al., 2004).

More definitely, comorbidity at the time of diagnosis 
is an independent prominent factor for survival among breast cancer patients (Patterson et al., 2010). The study showed that the presence of one chronic condition was comparable to one tumor stage modification in terms of breast cancer survival decrease (Patnaik et al., 2011). In Korea, breast cancer screening is recommended every year for women aged 40 years and above free of charge.

However, varying results have been reported regarding adherence to recommended screening among patients suffering from chronic diseases. Some chronic conditions were generally associated with higher cancer screening rates (e.g. cancer survivors, hypertension) (Constantinou et al., 2016; Heflin et al., 2002; Khan et al., 2008). However, some conditions were associated with lower cancer screening rates (e.g. diabetes, obesity, depression) (Constantinou et al., 2016; Lipscombe et al., 2005; Ludman et al., 2010; Martinez-Huedo et al., 2012; Zhao et al., 2009). Additionally, some chronic diseases including rheumatoid arthritis reported contradictory results in association with cancer screening (Kim et al., 2012; Schneeweiss et al., 2011).

When the overall effect of chronic morbidity breast cancer screening is studied using summary measures, increased comorbidity is associated with decreased screening in clinic based studies and with increased screening in population based studies (Heflin et al., 2002; Kiefe et al., 1998). Evidence on screening determinants is now extensive (Jepson et al., 2000) and a large number of variables are associated with mammography including demographic, socioeconomic characteristics and health behaviors (Moser et al., 2009).

In this context, the objective of our study was to identify chronic diseases that affect on the participation in breast cancer screening after adjustment of other determinants by using nationwide survey data in Korea.

\section{Materials and Methods}

\section{Data source}

This study was a cross sectional and population based study, the data came from the Korean National Health and Nutrition Examination Survey (KNHANES) 2012. The KNHANES 2012 has been chosen for the reason that it has the recent full information about chronic diseases as well as about breast and cervical cancer screening. Many government organizations and researchers used mostly KNHANES data. KNHANES is a continuing national surveillance system that evaluates the health and nutritional status of Koreans, monitors inclination in health risk factors and the prevalence of major chronic diseases and offers data for the development and evaluation of health policies and programs in Korea. KNHANES has been led in 1998, 2001, 2005, 2007-2009, 2010-2012 and 2013-2015, 2016 was the initial of the seventh KNHANES (2016-12018). The observation system of KNHANES has been managed by Korea Center for Disease Control and Prevention (KCDC). KNHANES is composed of three surveys: a health interview, health examination and nutrition survey (Kweon et al., 2014).

\section{Outcome}

The outcome was adherence to breast cancer screening, having undergone a mammography for women aged 40 years and above. The selection process for the studied samples is presented in figure 1. Our study was focusing on eight (8) chronic diseases: hypertension, diabetes, dyslipidemia, stroke, asthma, osteoarthritis, depression and cancer (other than breast cancer) that have been assessed during health examination and health interview in KNHANES V. Cancers focused on were gastric, liver, colon, breast, cervical, lung, thyroid cancer and others.

\section{Covariates}

To investigate whether the association between chronic conditions and screening participation was modified by the major screening determinants. The determinants included socioeconomic factors (age, education, marital status, average monthly house income and subjective health status) and health behaviors (alcohol drinking and smoking).

\section{Statistical analyses}

The analysis has accounted for the survey weighting. The general characteristics of participants were calculated using summary statistics. We compared screening participation between women with chronic diseases of interest versus women without the diseases in general using chi-squared test. Logistic regression analyses were conducted to estimate the difference between women with chronic diseases and women without chronic diseases who undergone breast cancer screening. To determine the variables that were independent predictors of breast cancer screening among women with chronic diseases; multivariate logistic regression was applied. All models were systematically adjusted for two categories of screening determinants: socioeconomic factors and health behaviors in a full adjusted model. The odds ratio (OR) and 95\% confidence interval (CI) were calculated to measure the strength of association. The statistical significance was considered to be $<0.05$. For all models, adherence to screening was the dependent variable and chronic conditions were specified as dichotomous explanatory variables. Available sampling design and overall non-response were applied and our estimates can be extrapolated to the total non-institutionalized civilian population of Korea. All statistical analyses were performed using Stata version 14 (Stata Corp LP, College Station, TX) software in survey mode.

\section{Results}

Associated general characteristics of participants with breast cancer screening

Overall, high participation in breast cancer screening was observed in women aged $40-59$ years $(76.8 \%)$. Women with education level of high school $(78.9 \%)$ were more likely to participate in breast cancer screening. Married women $(78.3 \%)$ were more likely to participate in breast cancer screening. Women with average monthly house income over four million won were more likely $(81.4 \%)$ to participate in breast cancer screening. Women who were never smokers $(75.9 \%)$ showed high participation 
DOI:10.31557/APJCP.2019.20.1.207

Breast Cancer Screening and Chronic Diseases

Table 1. Breast Cancer Screening Participation Rate According to General Characteristics and Presence of Chronic Disease

\begin{tabular}{|c|c|c|c|c|}
\hline & \multicolumn{4}{|c|}{ Breast cancer screening participation } \\
\hline & Total N (\%) & No N (\%) & Yes N (\%) & p-value* \\
\hline Age & & & & 0.0214 \\
\hline $40-59$ years & $1,218(100)$ & $237(23.21)$ & $981(76.79)$ & \\
\hline 60 years and over & $1,186(100)$ & $308(29.25)$ & $878(70.75)$ & \\
\hline Education & & & & $<0.001$ \\
\hline Lower than middle school & $1,357(100)$ & $306(23.42)$ & $1,051(76.58)$ & \\
\hline High school & $621(100)$ & $109(21.15)$ & $512(78.85)$ & \\
\hline College and over & $426(100)$ & $130(37.89)$ & $296(62.14)$ & \\
\hline Marital status & & & & $<0.001$ \\
\hline Married & $1,729(100)$ & $311(21.66)$ & $1,418(78.34)$ & \\
\hline Separated/Divorced/widowed & $648(100)$ & $227(36.63)$ & $421(63.37)$ & \\
\hline Singles & $27(100)$ & $7(30.34)$ & $20(69.66)$ & \\
\hline Average monthly house income & & & & 0.0004 \\
\hline Lower to 2 million won & $1,029(100)$ & $285(29.26)$ & $744(70.74)$ & \\
\hline 2 - 4 million won & $692(100)$ & $148(27.03)$ & $544(72.97)$ & \\
\hline Over 4 million won & $683(100)$ & $112(18.56)$ & $571(81.44)$ & \\
\hline Subjective health status & & & & 0.6295 \\
\hline $\mathrm{Bad}$ & $597(100)$ & $144(26.12)$ & $453(73.88)$ & \\
\hline Average & $1,242(100)$ & $284(26.04)$ & $958(73.96)$ & \\
\hline Good & $565(100)$ & $117(23.4)$ & $448(76.6)$ & \\
\hline Alcohol drinking & & & & $<0.001$ \\
\hline No & $713(100)$ & $221(34.99)$ & $492(65.01)$ & \\
\hline Yes & $1,691(100)$ & $324(21.97)$ & $1,367(78.03)$ & \\
\hline Smoking status & & & & 0.0057 \\
\hline Never & $2,231(100)$ & $482(24.07)$ & $1,749(75.93)$ & \\
\hline Past smoker & $84(100)$ & $29(37.43)$ & $55(62.57)$ & \\
\hline Intermittent/ Daily smoker & $89(100)$ & $34(39.84)$ & $55(60.16)$ & \\
\hline Presence of chronic disease & & & & $<0.001$ \\
\hline No & $875(100)$ & $236(30.64)$ & $639(69.36)$ & \\
\hline Yes & $1,529(100)$ & $309(21.8)$ & $1220(78.2)$ & \\
\hline
\end{tabular}

*, Chi-square test; N, Total number; \%, percentage; <, lower to.

Table 2. Logistic Regression for Breast Cancer Screening Participation Rate Associated with Diagnosis of Chronic Diseases and Other Factors

\begin{tabular}{lcccccc}
\hline Ever diagnosed as & \multicolumn{2}{c}{ Univariate analysis } & \multicolumn{2}{c}{ Multivariate analysis } & \multicolumn{2}{c}{ Multivariate adjusted for general characteristics } \\
& OR & $95 \%$ CI & OR & $95 \%$ CI & OR & $95 \%$ CI \\
\hline Hypertension & 1.23 & $0.94-1.62$ & 1.18 & $0.88-1.57$ & 1.15 & $0.82-1.62$ \\
Dyslipidemia & 1.87 & $1.33-2.62$ & 2.03 & $1.47-2.80$ & 1.85 & $1.27-2.69$ \\
Stroke & 0.65 & $0.33-1.30$ & 0.59 & $0.31-1.11$ & 0.69 & $0.34-1.39$ \\
Osteoarthritis & 1.49 & $1.12-1.98$ & 1.42 & $1.07-1.89$ & 1.42 & $1.31-2.06$ \\
Asthma & 0.52 & $0.29-0.92$ & 0.52 & $0.29-0.94$ & 0.44 & $0.24-0.82$ \\
Diabetes mellitus & 0.6 & $0.43-0.85$ & 0.44 & $0.31-0.63$ & 0.47 & $0.31-0.72$ \\
Depression & 1.17 & $1.12-1.71$ & 1.21 & $0.90-1.64$ & 1 & $0.68-1.46$ \\
Cancers & 3.4 & $1.57-7.35$ & 3.19 & $1.45-7.00$ & 2.7 & $1.26-5.79$ \\
\hline
\end{tabular}

OR, odd ratio; 95\% CI, 95\% confidence interval; Cancers, Other cancers than breast cancer (gastric, liver, colon, cervical, lung, thyroid and other cancers); General characteristics, Age, education, marital status, average monthly house income, subjective health, drinking experience and smoking status; Multivariate, (hypertension, dyslipidemia, stroke, osteoarthritis, asthma, diabetes mellitus, depression and cancers); Full adjusted, All covariates (general characteristics); Reference category is women who have not reported the disease of interest. 


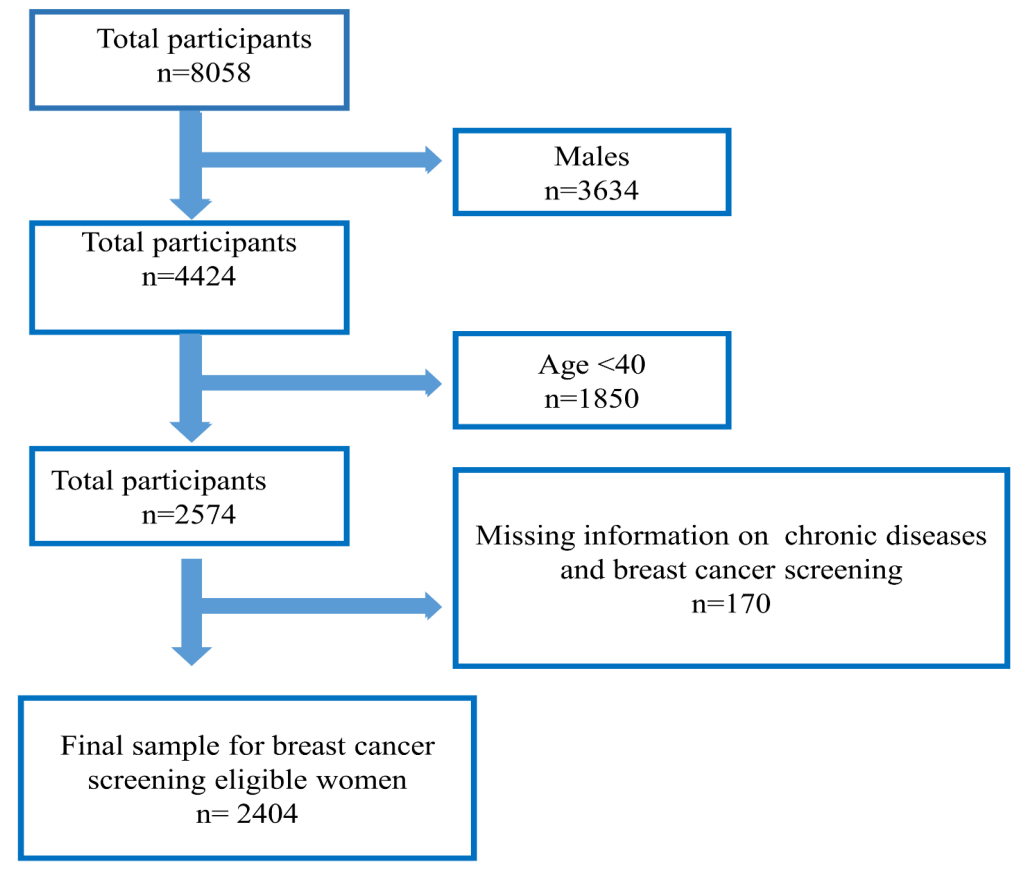

Figure 1. Flowchart Describing Breast Cancer Screening Sample Selection

in breast cancer screening. Women who have had any chronic disease (78.2\%) showed high participation in breast cancer screening. (Table 1).

About specific chronic conditions, hypertension $(28.5 \%)$ was the most prevalent in our study. For other diseases, their prevalence was $15.5 \%, 1.9 \%, 23 \%$, $4.3 \%, 9.1 \%, 21 \%$ and $3.8 \%$ for dyslipidemia, stroke, osteoarthritis, asthma, diabetes mellitus, depression and cancer, respectively (Figure 2).

\section{Uptake of mammography}

Overall, women who have ever been diagnosed as dyslipidemia $(\mathrm{OR}=1.85,95 \% \mathrm{CI}=1.27-2.69)$, osteoarthritis $(\mathrm{OR}=1.42,95 \% \mathrm{CI}=1.31-2.06)$ and cancers $(\mathrm{OR}=2.70$, $95 \% \mathrm{CI}=1.26-5.79)$ were more likely to participate in breast cancer screening even in full model adjusted for general characteristics compared to women without those chronic diseases. Low participation in breast cancer screening was observed in women who have ever been diagnosed as asthma $(\mathrm{OR}=0.44,95 \% \mathrm{CI}=0.24-0.82)$ and diabetes mellitus $(\mathrm{OR}=0.47,95 \% \mathrm{CI}=0.31-0.72)$ (Table 2 ). Women on treatment of dyslipidemia were more likely to participate in breast cancer screening $(\mathrm{OR}=$ $1.37,95 \% \mathrm{CI}=1.42-2.14$ ) compared to women who were not on treatment of dyslipidemia. Also, low participation in breast cancer screening has been observed in women who were on treatment of stroke $(\mathrm{OR}=0.37,95 \% \mathrm{CI}=$ $0.14-0.97)$, asthma $(\mathrm{OR}=0.41,95 \% \mathrm{CI}=0.15-0.92)$ and diabetes mellitus $(\mathrm{OR}=0.48,95 \% \mathrm{CI}=0.31-0.74)$ compared to women who were not on treatment of those conditions (Table 3).

\section{Discussion}

Our study was conducted to examine the participation of women with chronic diseases in breast cancer screening

Table 3. Logistic Regression Results for Breast Cancer Screening Participation Rate Associated with Chronic Diseases on Treatment and Other Factors

\begin{tabular}{lcccccc}
\hline On treatment of & \multicolumn{2}{c}{ Univariate analysis } & \multicolumn{2}{c}{ Multivariate analysis } & \multicolumn{2}{c}{ Multivariate adjusted for general characteristics } \\
& OR & $95 \%$ CI & OR & $95 \%$ CI & OR & $95 \%$ CI \\
\hline Hypertension & 1.19 & $0.92-1.54$ & 1.27 & $0.98-1.65$ & 1.12 & $0.83-1.50$ \\
Dyslipidemia & 1.37 & $0.90-2.08$ & 1.45 & $0.97-2.17$ & 1.37 & $1.42-2.14$ \\
Stroke & 0.37 & $0.14-0.97$ & 0.38 & $0.16-0.86$ & 0.36 & $0.16-0.85$ \\
Osteoarthritis & 1.13 & $0.75-1.71$ & 1.15 & $0.74-1.78$ & 1.07 & $0.66-1.72$ \\
Asthma & 0.41 & $0.15-0.92$ & 0.42 & $0.15-0.94$ & 0.41 & $0.16-1.09$ \\
Diabetes Mellitus & 0.6 & $0.42-0.87$ & 0.49 & $0.34-0.86$ & 0.48 & $0.31-0.74$ \\
Depression & 1.21 & $0.58-2.53$ & 1.32 & $0.65-2.67$ & 1.21 & $0.59-2.44$ \\
Cancers & 3.47 & $1.18-9.20$ & 3.62 & $1.18-8.18$ & 2.65 & $0.82-8.52$ \\
\hline
\end{tabular}

OR, odd ratio; 95\% CI, 95\% confidence interval; Cancers, Other cancers than breast cancer (gastric, liver, colon, cervical, lung, thyroid and other cancers); General characteristics, Age, education, marital status, average monthly house income, subjective health, drinking experience and smoking status; Multivariate, (hypertension, dyslipidemia, stroke, osteoarthritis, asthma, diabetes mellitus, depression and cancers); Full adjusted, All covariates (general characteristics); Reference category is women who have not reported the disease of interest. 


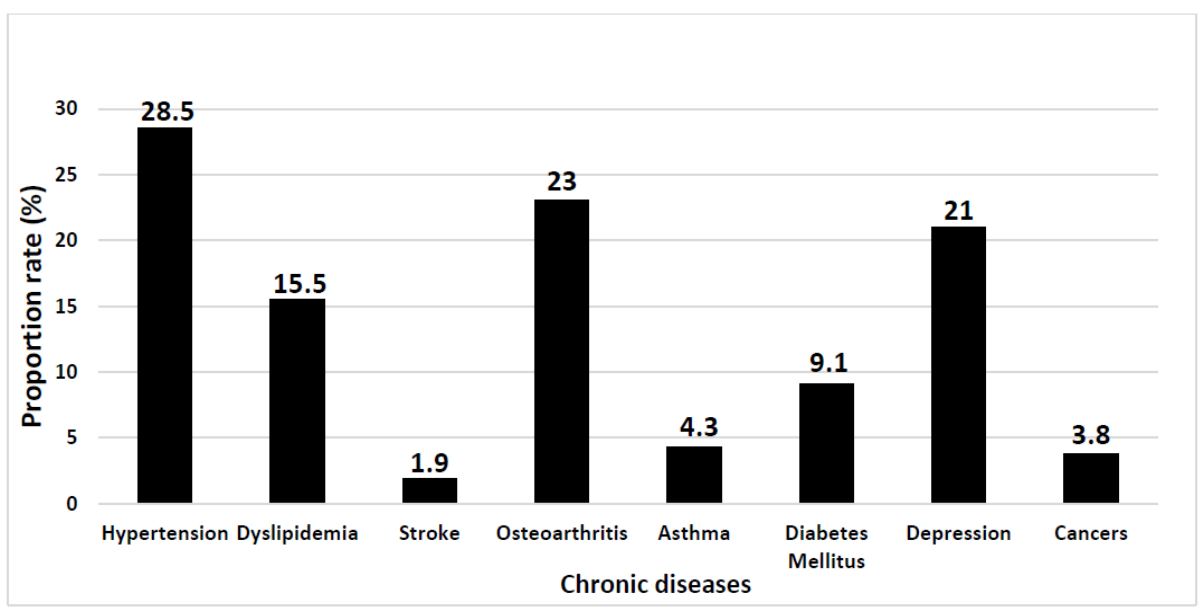

Figure 2. Ever Diagnosed as Chronic Disease in Women Aged of 40 Years and Over

using KNHANES data 2012. In this study, generally women with chronic diseases participated higher in breast cancer screening compared to women without chronic diseases. Adherence to breast cancer screening was higher in women who have ever been diagnosed as dyslipidemia, osteoarthritis, depression and cancer. The low participation in breast cancer screening was observed in women who have ever been diagnosed as asthma and diabetes mellitus. Based on treatment, high participation in breast cancer screening was observed in women who were on treatment of dyslipidemia and low participation in women who were on treatment of stroke, asthma and diabetes mellitus.

Being treated for a cancer will likely expose an individual to the awareness of breast cancer screening and the benefits associated with it. We also assume that cancer patients visit frequently health care facilities as they are worry that they can get another type of cancer so that can lead them to use more health services as well as breast cancer screening services. The higher participation in breast cancer screening of women with dyslipidemia may be due to easy accessibility of health services in Korea. Since elevated cholesterol level is asymptomatic and more unlikely to lead people to seek medical attention, its association with breast cancer screening is more likely mediated by health consciousness. People with dyslipidemia do not have disabilities and still use health care facilities as usually.

Patients with depression have high rates of somatization, symptom strengthening, more complain of nonspecific symptoms and sensitive awareness of bodily sensation that lead them to use more health care services as well as breast cancer screening services. Also, people with depression spend more time for counseling in health facilities, that can make them know more on preventive services as well as breast cancer screening.

Women who has ever diagnosed as osteoarthritis but had mild symptom still do not have significant barrier to receive breast cancer screening. However, when osteoarthritis needs to treat due to pain then the patients' movement to screening units can be limited. Thus women who ever diagnosed as osteoarthritis have relatively higher breast cancer screening rate, but women on treatment for osteoarthritis have lower participation in breast cancer screening.

Our study shows that women with diabetes mellitus tend to participate in breast cancer screening significantly lower both in ever diagnosed and on treatment than those without diabetes mellitus. The reason can be explained that more concentration of both patients and physicians is made on diabetes controlling rather than other preventive care practices including breast cancer screening.

Asthma is a condition that mostly found in women of families with lower income and low education level. Also screening rate in families with lower income as well as low education level is low. Women on treatment of stroke were associated with low participation in breast cancer screening, stroke cause serious disability so for people with stroke may not easily access health services due to the disability.

Socioeconomic status was associated with breast cancer screening. Women in age 40-59 were more likely to participate in breast cancer screening. Women with high school as education level were more likely to participate in breast cancer screening. Married women were more likely to participate in breast cancer screening. Women with average monthly house income more to four million were associated with high participation in breast cancer screening. Women who reported good health status were more likely to participate in breast cancer screening. Women who were no smoker were associated with high participation in breast cancer screening. Most chronic diseases were prevalent in women aged 60 years and above with low education level, living alone, with average monthly income lower to two million and women who reported that their health status was bad (results are not shown).

We did comparison of self-reported and directly measured prevalence of KNHANES 2012 for some prevalent chronic diseases (hypertension, dyslipidemia and diabetes mellitus) presented in KNHANES 2012 report (Results not shown). The difference observed in prevalence maybe due to recall bias of self-reported measures. In self-reported, it included the women who have ever been diagnosed as chronic diseases but by the time of directly measured they may not have the disease. 
The directly measured are taken at one point of time and some chronic diseases vary by time, also some chronic diseases may vary in measures depending on position (like hypertension), or depending on fasting status (like diabetes mellitus). The directly measured were not taken in hospital setting nor by physicians which could also cause the variation in measurement. The diagnosis of chronic diseases needs two or more direct measurements commonly. Thus the prevalence between self-reported and directly measured can be different.

\section{Strengths and limitations}

We used data from a large national survey, taking advantage of the survey's overall size to study a large number of chronic conditions and explanatory variables. This study is among few studies focusing on participation in breast cancer screening of women with chronic diseases in Korea. Self-reported measures of chronic diseases status and the receipt of cancer screening were used and are subject to recall bias. Nevertheless, some studies suggest that despite the fact that overestimation of adherence to cancer screening may occur, self-reporting may be the only effective and feasible way to gather data on preventive services uptake among large samples of the population (Howard et al., 2009). We were unable to exclude women who had already cancers or other conditions to be diagnosed therefore we were not sure whether a test was for screening only or for a diagnostic purpose. We were unable to exclude women for whom screening for cancers were no longer needed (e.g. women who had had a normal pap test for many years). It was unknown whether women attended screening through national cancer screening programs or private programs.

In conclusion, low participation in breast cancer screening was observed in women with diabetes mellitus, asthma and stroke. Higher participation in breast cancer screening has been found in women with dyslipidemia, depression, cancers and osteoarthritis. Socioeconomic characteristics and behavior factors were associated with breast and cervical cancer screening participation as well as with chronic diseases. The results from this study may provide an important contribution for helping to maintain and increase the participation in cancer screening of patients with chronic diseases. Educational programs targeted at the general population and healthcare professionals as well as women with chronic diseases to improve and maintain the awareness of the importance of cancer screening services need to be developed. To improve the participation rate for breast cancer screening, more attention should be given to women in lower sociodemographic groups. Further investigation is needed to better understand breast cancer screening among women with chronic diseases and to design interventions that efficiently increase screening coverage in these groups.

\section{Disclosure}

All authors declare no conflict of interest.

\section{Acknowledgements}

This study was supported by a grant (NCC 1610431) from Korean National Cancer Center.

\section{References}

Autier P, Boniol M, Gavin A, et al (2011). Breast cancer mortality in neighbouring European countries with different levels of screening but similar access to treatment: trend analysis of WHO mortality database. BMJ, 343, d4411.

Constantinou P, Dray-Spira R, Menvielle G (2016). Cervical and breast cancer screening participation for women with chronic conditions in France: results from a national health survey. BMC Cancer, 16, 255.

Ferlay J, Soerjomataram I, Dikshit R, et al (2015). Cancer incidence and mortality worldwide: sources, methods and major patterns in GLOBOCAN 2012. Int J Cancer, 136, 5.

Heflin MT, Oddone EZ, Pieper CF, et al (2002). The effect of comorbid illness on receipt of cancer screening by older people. J Am Geriatr Soc, 50, 1651-8.

Howard M, Agarwal G, Lytwyn A (2009). Accuracy of self-reports of Pap and mammography screening compared to medical record: a meta-analysis. Cancer Causes Control, 20, 1.

Jepson R, Clegg A, Forbes C, et al (2000). The determinants of screening uptake and interventions for increasing uptake: a systematic review. Health Technol Assess, 4, i-vii, 1-133.

Kerlikowske K, Grady D, Rubin SM, et al (1995). Efficacy of screening mammography. A meta-analysis. JAMA, 273, 149-54.

Kiefe CI, Funkhouser E, Fouad MN, et al (1998). Chronic disease as a barrier to breast and cervical cancer screening. J Gen Intern Med, 13, 357-65.

Khan NF, Ward A, Watson E, et al (2008). Long-term survivors of adult cancers and uptake of primary health services: a systematic review. Eur J Cancer, 44, 195-204.

Kim SC, Schneeweiss S, Myers JA, et al (2012). No differences in cancer screening rates in patients with rheumatoid arthritis compared to the general population. Arthritis Rheum, 64, 3076-82.

Kweon S, Kim Y, Jang MJ, et al (2014). Data resource profile: the Korea National Health and Nutrition Examination Survey (KNHANES). Int J Epidemiol, 43, 69-77.

La Vecchia C, Franceschi S, Decarli A, et al (1984). "Pap" smear and the risk of cervical neoplasia: quantitative estimates from a case-control study. Lancet, 2, 779-82.

Lipscombe LL, Hux JE, Booth GL (2005). Reduced screening mammography among women with diabetes. Arch Intern Med, 165, 2090-5.

Ludman EJ, Ichikawa LE, Simon GE, et al (2010). Breast and cervical cancer screening: specific effects of depression and obesity. Am J Prev Med, 38, 303-10.

Martinez-Huedo MA, de Andres AL, Hernandez-Barrera V, et al (2012). Adherence to breast and cervical cancer screening in Spanish women with diabetes: associated factors and trend between 2006 and 2010. Diabetes Metab, 38, 142-8.

Mendis S, Davis S, Norrving B (2015). Organizational update: the world health organization global status report on noncommunicable diseases 2014; one more landmark step in the combat against stroke and vascular disease. Stroke, 46, e121-2.

Moser K, Patnick J, Beral V (2009). Inequalities in reported use of breast and cervical screening in Great Britain: analysis of cross sectional survey data. $B M J, \mathbf{3 3 8}$, b2025.

Patnaik JL, Byers T, Diguiseppi C, et al (2011). The influence of comorbidities on overall survival among older women diagnosed with breast cancer. J Natl Cancer Inst, 103, 1101-11.

Patterson RE, Flatt SW, Saquib N, et al (2010). Medical 
comorbidities predict mortality in women with a history of early stage breast cancer. Breast Cancer Res Treat, 122, 859-65.

Piccirillo JF, Tierney RM, Costas I, et al (2004). Prognostic importance of comorbidity in a hospital-based cancer registry. JAMA, 291, 2441-7.

Sarfati D, Koczwara B, Jackson C (2016). The impact of comorbidity on cancer and its treatment. CA Cancer J Clin, 66, 337-50.

Schneeweiss SY, Meyers S, Liu JE, et al (2011). Cancer screening rates in patients with Rheumatoid Arthritis: No different than the general population. Arthritis Rheum, 63, 116.

Smith RA, Cokkinides V, Eyre HJ (2006). American Cancer Society guidelines for the early detection of cancer. $C A$ Cancer J Clin, 56, 11-25; 49-50.

Tabar L, Vitak B, Chen TH, et al (2011). Swedish two-county trial: impact of mammographic screening on breast cancer mortality during 3 decades. Radiology, 260, 658-63.

Wild CP (2012). The role of cancer research in noncommunicable disease control. J Natl Cancer Inst, 104, 1051-8.

Zhao G, Ford ES, Ahluwalia IB, et al (2009). Prevalence and trends of receipt of cancer screenings among US women with diagnosed diabetes. J Gen Intern Med, 24, 270-5.

\section{(c) (1) (9)}

This work is licensed under a Creative Commons AttributionNon Commercial 4.0 International License. 